\title{
Probabilistic solutions for a class of deterministic optimal allocation problems
}

\author{
Ka Chun Cheung* Jan Dhaene ${ }^{\dagger} \quad$ Yian Rong ${ }^{\ddagger}$ \\ Sheung Chi Phillip Yam ${ }^{\S}$
}

\begin{abstract}
We revisit the general problem of minimizing a separable convex function with both a budget constraint and a set of box constraints. This optimization problem arises naturally in many resource allocation problems in engineering, economics, finance and insurance. Existing literature tackles this problem by using the traditional Kuhn-Tucker theory, which leads to either iterative schemes or yields explicit solutions only under some special classes of convex functions owe to the presence of box constraints. This paper presents a novel approach of solving this constrained minimization problem by using the theory of comonotonicity. The key step is to apply an integral representation result to express each convex function as the stop-loss transform of some suitable random variable. By using this approach, we can derive and characterize not only the explicit solution, but also obtain its geometric meaning and some other qualitative properties.
\end{abstract}

Keywords: Optimal allocation, Constrained optimization, Comonotonicity, Stop-loss transform

$M S C:$ 90B99, 90C30

\section{Introduction and problem formulation}

Let $\left(X_{1}, \ldots, X_{n}\right)$ be the portfolio of risks (i.e., random variables representing losses) we are facing. A provision of $d$ dollars is available to be allocated among these $n$ risks. We use the function $f_{i}\left(d_{i}\right)$ to model the level of riskiness of the risk $X_{i}$ if $d_{i}$ dollars is allocated to $X_{i}$. When more capital is allocated to risk $i$, the position is considered to be safer, and hence the corresponding risk level is less. This means that $f_{i}$ should be a decreasing function. It is also natural to assume that the decrement is diminishing per unit of growth. Accordingly, $f_{i}$ is both decreasing and convex. As a typical example, we may take $f_{i}\left(d_{i}\right):=\rho\left(\left(X_{i}-d_{i}\right)_{+}\right)$, where $\rho$ is some convex and increasing functional. The amount $\rho\left(\left(X_{i}-d_{i}\right)_{+}\right)$could be

\footnotetext{
*The University of Hong Kong, Hong Kong. Email: kccg@hku.hk

${ }^{\dagger}$ KU Leuven, Leuven, Belgium. Email: Jan.Dhaene@econ.kuleuven.be

†The University of Hong Kong, Hong Kong. Email: yrong@hku.hk

$\S$ The Chinese University of Hong Kong. Email: scpyam@sta.cuhk.edu.hk
} 
interpreted for example as the provision required by the residual risk $\left(X_{i}-d_{i}\right)_{+}$after $d_{i}$ dollars has been allocated to the potential loss $X_{i}$.

Alternatively, instead of treating $f_{i}$ as a measurement of risk, we can think of it as a penalty function in the sense that allocated capital is expected to be as close as possible to the loss being allocated to, and that $f_{i}$ penalizes the deviation. This point of view was adopted by Zaks et al. (2006) and Frostig et al. (2007) in determining the fair price of a heterogeneous portfolio. Common examples of penalty functions include quadratic deviations and absolute deviations. With this interpretation, it is natural to assume that $f_{i}$ is convex, but not necessarily decreasing.

The considerations above lead us to study the minimization of the total required provision or total penalty by determining the optimal amount $d_{i}^{*}$ needed for each risk:

$$
\min _{d_{1}+\cdots+d_{n}=d} \sum_{i=1}^{n} f_{i}\left(d_{i}\right) .
$$

In the case of provision allocations and many other, it is common to require, apart from $d_{1}+\cdots+d_{n}=d$, that each allocation $d_{i}$ is positive. This consideration leads to formulate the following general problem:

$$
\min _{\left(d_{1}, \ldots, d_{n}\right) \in \mathcal{A}(d)} \sum_{i=1}^{n} f_{i}\left(d_{i}\right),
$$

where the set of admissible allocations equals

$$
\mathcal{A}(d)=\left\{\left(d_{1}, \ldots, d_{n}\right) \in \mathbb{R}^{n} \mid d_{1}+\cdots+d_{n}=d, d_{i} \in\left[l_{i}, u_{i}\right], i=1, \ldots, n\right\},
$$

in where $l_{i}, u_{i}$ are given fixed constants with $l_{i}<u_{i}$. In other words, $\mathcal{A}(d)$ is the intersection of the hyperplane $\left\{\left(x_{1}, \ldots, x_{n}\right) \in \mathbb{R}^{n} \mid x_{1}+\cdots+x_{n}=d\right\}$ and the $n$-dimensional box $\prod_{i=1}^{n}\left[l_{i}, u_{i}\right]$. The constraints $l_{i} \leq d_{i} \leq u_{i}$ are commonly referred to as box constraints. To ensure that $\mathcal{A}(d)$ is non-empty and is not a singleton, we assume that

$$
l_{1}+\cdots+l_{n}<d<u_{1}+\cdots+u_{n} .
$$

Throughout this paper, we assume that each $f_{i}$ is convex and continuous on $\left[l_{i}, u_{i}\right]$, but is not necessarily decreasing. For simplicity, we also assume that $\left(f_{i}\right)_{+}^{\prime}\left(l_{i}\right)$ and $\left(f_{i}\right)_{-}^{\prime}\left(u_{i}\right)$ are finite for all $i$, where $f_{+}^{\prime}$ and $f_{-}^{\prime}$ denote the right-hand and left-hand derivative of any convex function $f$. Since $\mathcal{A}(d)$ is non-empty and compact and the objective function is continuous, a solution always exists.

The linear constraint $d_{1}+\cdots+d_{n}=d$ can easily be extended to a more general linear constraint of the form

$$
c_{1} d_{1}+\cdots+c_{n} d_{n}=d
$$

where $c_{1}, \ldots, c_{n}$ are some fixed constants. We may assume that all of them are non-zero: if $c_{i}=0$ for some $i$, we can minimize $f_{i}\left(d_{i}\right)$ over $d_{i} \in\left[l_{i}, u_{i}\right]$ separately as a one-dimensional problem. With this new constraint, we have the following more general minimization problem:

$$
\min _{c_{1} d_{1}+\cdots+c_{n} d_{n}=d, l_{i} \leq d_{i} \leq u_{i}} \sum_{i=1}^{n} f_{i}\left(d_{i}\right) .
$$


Assume that the admissible set is non-empty. Let $\tilde{d}_{i}:=c_{i} d_{i}$ and $\tilde{f}_{i}(x):=f_{i}\left(x / c_{i}\right)$ for all $i$, then Problem (3) becomes

$$
\min _{\tilde{d}_{1}+\cdots+\tilde{d}_{n}=d, \tilde{d}_{i} \in\left[\tilde{l}_{i}, \tilde{u}_{i}\right]} \sum_{i=1}^{n} \tilde{f}_{i}\left(\tilde{d}_{i}\right),
$$

where $\left[\tilde{l}_{i}, \tilde{u}_{i}\right]=\left[c l_{i}, c u_{i}\right]$ if $c_{i}>0$, and $\left[\tilde{l}_{i}, \tilde{u}_{i}\right]=\left[c u_{i}, c l_{i}\right]$ if $c_{i}<0$. With this transformation, Problem (3) can be treated as a special case of Problem (1).

Capital allocation rules in the form of (1) or (3) are fairly general. It covers the various optimization-based models proposed and studied in Dhaene et al. (2012) and Zaks (2013), and also the insurance pricing models studied in Zaks et al. (2006) and Frostig et al. (2007). These problems also arise naturally in many optimization models in economics, operation management, finance, marketing, etc. We refer to Bitran and Hax (1981), Luss and Gupta (1975), Stefanov (2005) and the references therein, for various applications and discussions. Existing solution methods include standard convex programming (Kuhn-Tucker theory), dynamic programming (for instance, Wilkinson and Gupta (1969)) and the iterative method (e.g. Luss and Gupta (1975), Stefanov (2005)). The classical Kuhn-Tucker theory lies at the heart of all these different approaches. Similar allocation problems for future not-yetrealized risk or payoff, rather than for the current deterministic capital in the current context, has also gained considerable attention in recent years, we refer to Rüschendorf (2013) for a comprehensive discussion.

If the box constraints $d_{i} \in\left[l_{i}, u_{i}\right]$ are removed, and each $f_{i}$ is defined on the whole real line, Problem (1) is just the classical "infimum-convolution" of the convex functions $f_{1}, \ldots, f_{n}$ in convex analysis, which is well-studied in the literature (see, for instance, Rockafellar (1970)). The introduction of the box constraints $d_{i} \in\left[l_{i}, u_{i}\right]$, which consist of a total of $2 n$ onesided inequality constraints, makes the problem more difficult and possibly non-tractable analytically.

This paper presents an alternative method to solve Problem (1). Instead of using the traditional Lagrangian technique, we first express each convex function $f_{i}$ as the stop-loss transform of some random variable, so that the objective function becomes a sum of stop-loss transform. The box constraints can be effectively captured and removed by carefully choosing the random variables. Problem (1) then becomes the minimization of a sum of stop-loss transform subject to a homogeneous linear constraint. We demonstrate how the theory of comonotonicity can be used to solve such minimization problem effectively, with the solution set being completely characterized and explicitly expressed in an intuitive geometric way. While the transformation of convex functions into stop-loss transforms is not new and has been applied in different areas, its application in optimization has not been explored in the literature except in the recent paper Cheung et al. (2014). Further advantages of this approach are that we can easily obtain various qualitative properties of solutions of Problem (1), such as their uniqueness and conditions where the box constraints are binding; furthermore, some well-known results in convex analysis associated with infimum-convolution can be derived easily.

This paper is organized as follows. Section 2 reviews basic properties of the notion of comonotonicity. Special focus is put on the geometry of the support of a comonotonic random vector; in particular, we study how the support of a comonotonic random vector intersects with a given hyperplane. Section 3 studies a special case of Problem (1), in which each $f_{i}$ is a stop- 
loss transform. We indicate how Problem (1) can be solved using simple geometric arguments and the results in Section 2. In Section 4, we explain how one can express a given convex function as the stop-loss transform of some random variable. Section 5 combines the ideas in Sections 3 and 4 together to present a novel way to solve Problem (1). We not only provide a complete and explicit characterization of the solutions to Problem (1), but also derive some qualitative properties of the solutions. In Section 6, we give examples to demonstrate how the theory can be applied. Section 7 indicates several variants of Problem (1) to illustrate the flexibility of our model. Finally, Section 8 discusses how the techniques can be used when the box constraints are removed, in which case Problem (1) is related to the notion of infimum-convolution in convex analysis.

\section{Supports of comonotonic random vectors}

This section reviews some properties of comonotonicity that are pertinent to our later analysis. The focus here is on the behavior of the support of a comonotonic random vector, and how it intersects with a hyperplane with normal 1. In fact, it will be shown in Section 5 that the solution of Problem (1) can always be expressed as an intersection of such kind.

Following Kaas et al. (2000), a set $A \subset \mathbb{R}^{n}$ is said to be comonotonic if any two points in $A$ can be ordered componentwise, that is, for any $\left(x_{1}, \ldots, x_{n}\right)$ and $\left(y_{1}, \ldots, y_{n}\right)$ in $A,\left(x_{i}-y_{i}\right)\left(x_{j}-\right.$ $\left.y_{j}\right) \geq 0$ for any $i, j \in\{1, \ldots, n\}$. A random vector $\left(X_{1}, \ldots, X_{n}\right)$ is said to be comonotonic if there is some comonotonic set $A \subset \mathbb{R}^{n}$ so that $\mathbb{P}\left(\left(X_{1}, \ldots, X_{n}\right) \in A\right)=1$. Comonotonicity of $\left(X_{1}, \ldots, X_{n}\right)$ is equivalent to $\left(X_{1}, \ldots, X_{n}\right) \stackrel{d}{=}\left(F_{X_{1}}^{-1}(U), \ldots, F_{X_{n}}^{-1}(U)\right)$ for any uniform $(0,1)$ random variable $U$. In this paper, for any given random vector $\mathbf{X}=\left(X_{1}, \ldots, X_{n}\right)$, we define its comonotonic modification $\mathbf{X}^{c}=\left(X_{1}^{c}, \ldots, X_{n}^{c}\right)$ as $\mathbf{X}^{c}:=\left(F_{X_{1}}^{-1}(U), \ldots, F_{X_{n}}^{-1}(U)\right)$, where $U$ is an arbitrary uniform $(0,1)$ random variable. By construction, a comonotonic modification is always comonotonic and has the same marginal distributions as the original random vector. For a comprehensive overview of the theory on comonotonicity, we refer to Dhaene et al. (2002).

Throughout this paper, $F_{X}^{-1}$ denotes the left-continuous inverse of the distribution function $F_{X}$ of any random variable $X$ :

$$
F_{X}^{-1}(p):=\inf \left\{x \in \mathbb{R} \mid F_{X}(x) \geq p\right\}, \quad 0 \leq p \leq 1 .
$$

Similarly, the right-continuous inverse distribution function is defined as

$$
F_{X}^{-1+}(p):=\sup \left\{x \in \mathbb{R} \mid F_{X}(x) \leq p\right\}, \quad 0 \leq p \leq 1 .
$$

With the convention that $\inf \emptyset=+\infty$ and $\sup \emptyset=-\infty, F_{X}^{-1+}(0)$ and $F_{X}^{-1}(1)$ are the essential infimum and essential supremum of $X$ respectively. By definition, $F_{X}^{-1}(0)=-\infty$ and $F_{X}^{-1+}(1)=\infty$ regardless of the actual distribution of $X$. For our later purpose, we also need the notion of $\alpha$-mixed inverse distribution function. Following Kaas et al. (2000), it is defined as

$$
F_{X}^{-1(\alpha)}(p):=\alpha F_{X}^{-1}(p)+(1-\alpha) F_{X}^{-1+}(p), \quad 0 \leq p \leq 1,0 \leq \alpha \leq 1 .
$$

For consistency, we also adopt the convention $0 \cdot \pm \infty=0$ so that $F_{X}^{-1(0)}(p)=F_{X}^{-1+}(p)$ and $F_{X}^{-1(1)}(p)=F_{X}^{-1}(p)$ for any $0 \leq p \leq 1$. 
In the remainder of this section, $\mathbf{X}^{c}=\left(X_{1}^{c}, \ldots, X_{n}^{c}\right)$ denotes a fixed comonotonic random vector with marginal distribution functions $F_{1}, \ldots, F_{n}$, and $S^{c}$ is the comonotonic sum $X_{1}^{c}+$ $\cdots+X_{n}^{c}$. A fundamental property of comonotonicity (see for instance Denneberg (1994) or Dhaene et al. (2002)) is that the inverse distribution function of $S^{c}$ can be computed explicitly as follows:

$$
F_{S^{c}}^{-1(\alpha)}(p)=F_{1}^{-1(\alpha)}(p)+\cdots+F_{n}^{-1(\alpha)}(p), \quad 0 \leq p \leq 1,0 \leq \alpha \leq 1 .
$$

By definition, it is clear that

$$
\operatorname{msupp}\left(\mathbf{X}^{c}\right):=\left\{\left(F_{1}^{-1}(u), \ldots, F_{n}^{-1}(u)\right) \mid 0<u<1\right\}
$$

is a comonotonic set in $\mathbb{R}^{n}$ and is a support ${ }^{1}$ of $\mathbf{X}^{c}$. We say that $\mathbf{s} \in \mathbb{R}^{n}$ is a comonotonic support point of $\mathbf{X}^{c}$, if $\{\mathbf{s}\} \cup \operatorname{msupp}\left(\mathbf{X}^{c}\right)$ is again comonotonic. In other words, adding a comonotonic support point to $\operatorname{msupp}\left(\mathbf{X}^{c}\right)$ will not destroy its comonotonicity. The collection of all comonotonic support points of $\mathbf{X}^{c}$ will be denoted as $\operatorname{csupp}\left(\mathbf{X}^{c}\right)$ :

$$
\operatorname{csupp}\left(\mathbf{X}^{c}\right):=\left\{\mathbf{s} \in \mathbb{R}^{n} \mid\{\mathbf{s}\} \cup \operatorname{msupp}\left(\mathbf{X}^{c}\right) \text { is comonotonic }\right\} .
$$

Obviously, $\operatorname{msupp}\left(\mathbf{X}^{c}\right) \subset \operatorname{csupp}\left(\mathbf{X}^{c}\right)$, and $\operatorname{msupp}\left(\mathbf{X}^{c}\right) \cup\{\mathbf{s}\}$ is also a comonotonic support of $\mathbf{X}^{c}$ for every $\mathbf{s} \in \operatorname{csupp}\left(\mathbf{X}^{c}\right)$. However, $\operatorname{csupp}\left(\mathbf{X}^{c}\right)$ is not necessarily comonotonic.

For any $d \in \mathbb{R}$, we denote by $\ell(d)$ the hyperplane

$$
\left\{\mathbf{d}=\left(d_{1}, \ldots, d_{n}\right) \in \mathbb{R}^{n} \mid d_{1}+\cdots+d_{n}=d\right\} .
$$

As indicated earlier, we are interested in finding the intersection $\ell(d) \cap \operatorname{csupp}\left(\mathbf{X}^{c}\right)$, which will be denoted as $i\left(d, \mathbf{X}^{c}\right)$ :

$$
i\left(d, \mathbf{X}^{c}\right):=\left\{\mathbf{s} \in \mathbb{R}^{n} \mid\{\mathbf{s}\} \cup \operatorname{msupp}\left(\mathbf{X}^{c}\right) \text { is comonotonic and } s_{1}+\cdots+s_{n}=d\right\} .
$$

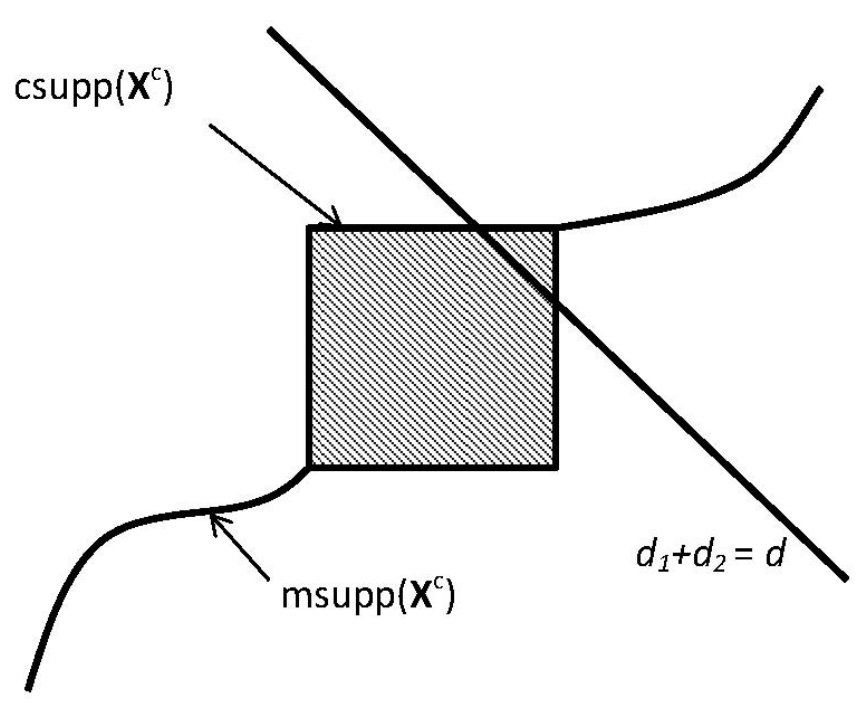

Figure 1: The set $i\left(d, \mathbf{X}^{c}\right)$ is the portion of the line $d_{1}+d_{2}=d$ that lies inside $\operatorname{csupp}\left(\mathbf{X}^{c}\right)$.

\footnotetext{
${ }^{1}$ By a support of a random variable or a random vector $Y$, we mean any Borel measurable set $A$ such that $\mathbb{P}(Y \in A)=1$.
} 
First, we observe that if $d<F_{S^{c}}^{-1+}(0)$ or $d>F_{S^{c}}^{-1}(1)$, the cardinality of $i\left(d, \mathbf{X}^{c}\right)$ is infinity. For if $d<F_{S^{c}}^{-1+}(0)=F_{1}^{-1+}(0)+\cdots+F_{n}^{-1+}(0)$, there are infinitely many possible ways to decompose $d$ into a sum $d_{1}+\cdots+d_{n}$ such that $d_{i} \leq F_{i}^{-1+}(0)$ for each $i$. Such a decomposition belongs to $i\left(d, \mathbf{X}^{c}\right)$ by definition. The case for $d>F_{S^{c}}^{-1}(1)$ is similar.

The following result characterizes the set $i\left(d, \mathbf{X}^{c}\right)$ for the more interesting and relevant case where $F_{S^{c}}^{-1+}(0) \leq d \leq F_{S^{c}}^{-1}(1)$. Its geometric meaning is clear and is described in Figure 1: $i\left(d, \mathbf{X}^{c}\right)$ is simply the portion of the hyperplane $\ell(d)$ that lies inside the box of comonotonic support points.

Proposition 2.1. Suppose that $d$ is a real number such that $F_{S^{c}}^{-1+}(0) \leq d \leq F_{S^{c}}^{-1}(1)$. Then

$$
i\left(d, \mathbf{X}^{c}\right)=\left\{\left(d_{1}, \ldots, d_{n}\right) \in \ell(d) \mid F_{i}^{-1}\left(F_{S^{c}}(d)\right) \leq d_{i} \leq F_{i}^{-1+}\left(F_{S^{c}}(d)\right) \text { for all } i\right\} .
$$

Proof: We first assume that $\sum_{i=1}^{n} F_{i}^{-1+}(0)<d<\sum_{i=1}^{n} F_{i}^{-1}(1)$. In this case, $0<F_{S^{c}}(d)<1$. Suppose $\mathbf{d}=\left(d_{1}, \ldots, d_{n}\right)$ belongs to the set on the right hand side of $(5)$. Then for any $i=1, \ldots, n$, we have $d_{i} \geq F_{i}^{-1}(p)$ when $p \in\left(0, F_{S}(d)\right]$ and $d_{i} \leq F_{i}^{-1}(p)$ when $p \in\left(F_{S^{c}}(d), 1\right)$, so $\mathbf{d} \cup \operatorname{msupp}\left(\mathbf{X}^{c}\right)$ is comonotonic. Therefore, $\mathbf{d}$ is a comonotonic support point of $\mathbf{X}^{c}$ and hence it lies in $i\left(d, \mathbf{X}^{c}\right)$.

Now we suppose that $\mathbf{d}=\left(d_{1}, \ldots, d_{n}\right) \in i\left(d, \mathbf{X}^{c}\right)$. Notice that

$$
\sum_{i=1}^{n} F_{i}^{-1}\left(F_{S^{c}}(d)\right)=F_{S^{c}}^{-1}\left(F_{S^{c}}(d)\right) \leq d \leq F_{S^{c}}^{-1+}\left(F_{S^{c}}(d)\right)=\sum_{i=1}^{n} F_{i}^{-1+}\left(F_{S^{c}}(d)\right) .
$$

Since $\mathbf{d} \in \operatorname{csupp}\left(\mathbf{X}^{c}\right)$, either $F_{i}^{-1}\left(F_{S^{c}}(d)\right) \leq d_{i}$ for all $i$ or $F_{i}^{-1}\left(F_{S^{c}}(d)\right)>d_{i}$ for all $i$. The second possibility is ruled out by the second inequality in (6) and the condition that $\mathbf{d} \in \ell(d)$, unless $d_{i}=F_{i}^{-1}\left(F_{S^{c}}(d)\right)$ for all $i$. Therefore, $F_{i}^{-1}\left(F_{S^{c}}(d)\right) \leq d_{i}$ for all $i$. By the same argument, $d_{i} \leq F_{i}^{-1+}\left(F_{S^{c}}(d)\right)$ for all $i$. This proves the reverse inclusion.

If $d=F_{S^{c}}^{-1+}(0)=\sum_{i=1}^{n} F_{i}^{-1+}(0) \in \mathbb{R}$, the only way to decompose $d$ into a sum $d=d_{1}+\cdots+d_{n}$ in such a way that $\mathbf{d} \cup \operatorname{msupp}\left(\mathbf{X}^{c}\right)$ is comonotonic is given by $d=\sum_{i=1}^{n} F_{i}^{-1+}(0)$. In this case, $i\left(d, \mathbf{X}^{c}\right)=\left\{\left(F_{1}^{-1+}(0), \ldots, F_{n}^{-1+}(0)\right)\right\}$. If $F_{S^{c}}(d)=0$, the right hand side of $(5)$ becomes

$$
\left\{\left(d_{1}, \ldots, d_{n}\right) \in \mathbb{R}^{n} \mid d_{1}+\cdots+d_{n}=d,-\infty<d_{i} \leq F_{i}^{-1+}(0) \text { for all } i\right\},
$$

which contains $\left(F_{1}^{-1+}(0), \ldots, F_{n}^{-1+}(0)\right)$ only; if $F_{S^{c}}(d)>0$, the right hand side of $(5)$ becomes

$$
\left\{\left(d_{1}, \ldots, d_{n}\right) \in \mathbb{R}^{n} \mid d_{1}+\cdots+d_{n}=d, F_{i}^{-1+}(0) \leq d_{i} \leq F_{i}^{-1+}\left(F_{S^{c}}(d)\right) \text { for all } i\right\},
$$

again, this set contains $\left(F_{1}^{-1+}(0), \ldots, F_{n}^{-1+}(0)\right)$ only.

The proof for the case where $d=F_{S^{c}}^{-1}(1)=\sum_{i=1}^{n} F_{i}^{-1}(1) \in \mathbb{R}$ is similar and so it is omitted.

To study the cardinality $i\left(d, \mathbf{X}^{c}\right)$, we introduce the following subset of $\mathbb{R}$ :

$$
s\left(\mathbf{X}^{c}\right):=\left\{d \in \mathbb{R} \mid d=\sum_{i=1}^{n} F_{i}^{-1}(p), \quad p \in(0,1], \text { or } \quad d=\sum_{i=1}^{n} F_{i}^{-1+}(p), \quad p \in[0,1)\right\} .
$$

Corollary 2.2. Suppose that $d$ is a real number such that $F_{S^{c}}^{-1+}(0) \leq d \leq F_{S^{c}}^{-1}(1)$. If $d \in s\left(\mathbf{X}^{c}\right)$, then $\operatorname{card}\left(i\left(d, \mathbf{X}^{c}\right)\right)=1$; otherwise, if $d \notin s\left(\mathbf{X}^{c}\right)$, 
(a) $\operatorname{card}\left(i\left(d, \mathbf{X}^{c}\right)\right)=\infty$ if there are more than one of $F_{i}^{-1}, i=1, \ldots, n$, jump at $F_{S^{c}}(d)$;

(b) $\operatorname{card}\left(i\left(d, \mathbf{X}^{c}\right)\right)=1$ if exactly one of $F_{i}^{-1}, i=1, \ldots, n$, jumps at $F_{S^{c}}(d)$.

Proof: For the first assertion, consider $d \in s\left(\mathbf{X}^{c}\right)$, and suppose that $d=\sum_{i=1}^{n} F_{i}^{-1}(p)$ for some $p \in(0,1]$ (the argument for the case where $d=\sum_{i=1}^{n} F_{i}^{-1+}(p)$ for some $p \in[0,1)$ is similar). Obviously, $\left(F_{1}^{-1}(p), \ldots, F_{n}^{-1}(p)\right) \in i\left(d, \mathbf{X}^{c}\right)$. If $\mathbf{s}=\left(s_{1}, \ldots, s_{n}\right)$ is a different point in $i\left(d, \mathbf{X}^{c}\right)$, then by the definition of comonotonicity, either $F_{i}^{-1}(p) \leq s_{i}$ for all $i$, or $F_{i}^{-1}(p) \geq s_{i}$ for all $i$, with the inequality being strict for at least one $i$ in both possibilities. At the same time, it is required that $\sum_{i=1}^{n} F_{i}^{-1}(p)=\sum_{i=1}^{n} s_{i}=d$. Clearly, such a point $\mathbf{s}$ does not exist, so $i\left(d, \mathbf{X}^{c}\right)$ is a singleton.

For the second assertion, note that $d \notin s\left(\mathbf{X}^{c}\right)$ implies that $F_{S^{c}}^{-1+}(0)<d<F_{S^{c}}^{-1}(1)$, or equivalently $0<F_{S^{c}}(d)<1$. In this case, at least one of the $F_{i}^{-1}, i=1, \ldots, n$, jumps at $F_{S^{c}}(d)$. If not, $F_{i}^{-1}\left(F_{S^{c}}(d)\right)=F_{i}^{-1+}\left(F_{S^{c}}(d)\right)$ for all $i$, summing over $i$ yields $F_{S^{c}}^{-1}\left(F_{S^{c}}(d)\right)=$ $F_{S^{c}}^{-1+}\left(F_{S^{c}}(d)\right)$ and hence $d=F_{S^{c}}^{-1}\left(F_{S^{c}}(d)\right)=F_{1}^{-1}\left(F_{S^{c}}(d)\right)+\cdots+F_{n}^{-1}\left(F_{S^{c}}(d)\right)$, which contradicts the assumption that $d \notin s\left(\mathbf{X}^{c}\right)$. Now the result follows from (5).

Corollary 2.3. Suppose that $F_{S^{c}}^{-1+}(0)<d<F_{S^{c}}^{-1}(1)$. If we define $d_{i}^{*}=F_{i}^{-1(\alpha)}\left(F_{S^{c}}(d)\right)$ for $i=1, \ldots, n$, where $\alpha \in[0,1]$ is a solution of $F_{S^{c}}^{-1(\alpha)}\left(F_{S^{c}}(d)\right)=d$, then $\left(d_{1}^{*}, \ldots, d_{n}^{*}\right) \in i\left(d, \mathbf{X}^{c}\right)$.

Proof: This corollary follows from (5) and the fact that $F_{i}^{-1}\left(F_{S^{c}}(d)\right) \leq d_{i}^{*} \leq F_{i}^{-1+}\left(F_{S^{c}}(d)\right)$ for all $i$.

\section{A canonical optimal capital allocation problem}

Before solving Problem (1), we first consider the well known canonical special case where $f_{i}\left(d_{i}\right)$ takes the form of $\mathbb{E}\left[\left(X_{i}-d_{i}\right)_{+}\right]$for a given integrable random variable $X_{i}$ :

$$
\min _{d_{1}+\cdots+d_{n}=d} \sum_{i=1}^{n} \mathbb{E}\left[\left(X_{i}-d_{i}\right)_{+}\right] .
$$

Notice that $d_{1}+\cdots+d_{n}=d$ is the only constraint in Problem (7). We do not impose any box constraint to restrict the individual allocations. In the next two sections, we will demonstrate that Problem (1) with box constraints can always be transformed into the form of Problem (7) without box constraints. Therefore, it is instructive to review this problem and to understand how comonotonicity comes to play.

Later in this section, we consider a much more general version of this canonical allocation problem in which the expectation will be replaced by a general risk measure.

Using the notion of comonotonicity, Kaas et al. (2002) used a simple geometric argument to prove that for $\sum_{i=1}^{n} F_{X_{i}}^{-1+}(0)<d<\sum_{i=1}^{n} F_{X_{i}}^{-1}(1)$, a solution to Problem (7) is given by

$$
d_{i}^{*}=F_{X_{i}}^{-1(\alpha)}\left(F_{S^{c}}(d)\right), \quad i=1, \ldots, n,
$$

in which $\alpha \in[0,1]$ is a solution of the equation $F_{S^{c}}^{-1(\alpha)}\left(F_{S^{c}}(d)\right)=d$, and $S^{c}:=X_{1}^{c}+\cdots+X_{n}^{c}$ where $\mathbf{X}^{c}=\left(X_{1}^{c}, \ldots, X_{n}^{c}\right)$ is a comonotonic modification of $\left(X_{1}, \ldots, X_{n}\right)$. It is easy to see that 
this solution is indeed a comonotonic support point of $\mathbf{X}^{c}$, and hence it belongs to $i\left(d, \mathbf{X}^{c}\right)$. The following theorem gives a full characterization of the solution set of Problem (7) for any $d \in \mathbb{R}$ in terms of $i\left(d, \mathbf{X}^{c}\right)$, which covers (8), the result from Kaas et al. (2002), as a special case.

Theorem 3.1. For any $d \in \mathbb{R}$, the solution set of Problem (7) is $i\left(d, \mathbf{X}^{c}\right)$.

Proof: $\quad$ Let $U$ be uniform $(0,1), \mathbf{X}^{c}:=\left(F_{X_{1}}^{-1}(U), \ldots, F_{X_{n}}^{-1}(U)\right)$, and $S^{c}:=F_{X_{1}}^{-1}(U)+\cdots+$ $F_{X_{n}}^{-1}(U)$. First, we may replace each $X_{i}$ in Problem $(7)$ by $F_{X_{i}}^{-1}(U)$. For any $d_{1}+\cdots+d_{n}=d$, we have

$$
\sum_{i=1}^{n} \mathbb{E}\left[\left(F_{X_{i}}^{-1}(U)-d_{i}\right)_{+}\right] \geq \mathbb{E}\left[\left(\sum_{i=1}^{n} F_{X_{i}}^{-1}(U)-\sum_{i=1}^{n} d_{i}\right)_{+}\right]=\mathbb{E}\left[\left(S^{c}-d\right)_{+}\right],
$$

so $\mathbb{E}\left[\left(S^{c}-d\right)_{+}\right]$is a lower bound of the objective function in $(7)$.

For any $\mathbf{d}=\left(d_{1}, \ldots, d_{n}\right) \in i\left(d, \mathbf{X}^{c}\right),\{\mathbf{d}\} \cup \operatorname{msupp}\left(\mathbf{X}^{c}\right)$ is comonotonic by definition, so either $\left(F_{X_{i}}^{-1}(U)-d_{i}\right)_{+}=F_{X_{i}}^{-1}(U)-d_{i}$ for all $i$ simultaneously, or $\left(F_{X_{i}}^{-1}(U)-d_{i}\right)_{+}=0$ for all $i$ simultaneously. In either case, $\sum\left(F_{X_{i}}^{-1}(U)-d_{i}\right)_{+}=\left(S^{c}-d\right)_{+}$, so $\mathbf{d}$ is a solution of Problem (7).

Finally, suppose $\mathbf{d} \in \ell(d)$ but $\mathbf{d} \notin \operatorname{csupp}\left(\mathbf{X}^{c}\right)$. Then there exist some $i, j \in\{1, \ldots, n\}$ and $u \in(0,1)$ such that $\left(F_{X_{i}}^{-1}(u)-d_{i}\right)\left(F_{X_{j}}^{-1}(u)-d_{j}\right)<0$. By the left continuity of $F^{-1}$, this strict inequality continues to hold on $[u-\varepsilon, u]$ for some $\varepsilon>0$. Therefore, $\sum\left(F_{X_{i}}^{-1}(U)-d_{i}\right)_{+}>$ $\left(S^{c}-d\right)_{+}$with a strictly positive probability, and so $\mathbf{d}$ is not optimal.

We remark that similar results can be found in Chen et al. (2015), in which the authors discuss the issue of (non)-uniqueness of the solution of Problem (7). Without recourse to the geometric notion of $i\left(d, \mathbf{X}^{c}\right)$, they show directly that the solution set is given by $i\left(d, \mathbf{X}^{c}\right)$.

The following result is a direct consequence of Theorem 3.1, Corollary 2.2 and Corollary 2.3.

Corollary 3.2. If $d \in s\left(\mathbf{X}^{c}\right)$, then Problem (7) admits a unique solution, and if $F_{S^{c}}^{-1+}(0)<$ $d<F_{S^{c}}^{-1}(1)$, then any solution $\left(d_{1}^{*}, \ldots, d_{n}^{*}\right)$ of Problem (7) satisfies $F_{X_{i}}^{-1+}(0) \leq d_{i}^{*} \leq F_{X_{i}}^{-1}(1)$.

Upon examining first part of the proof of Theorem 3.1 carefully, one can see that $i\left(d, \mathbf{X}^{c}\right)$ is indeed not only the solution set of Problem (7), but is also contained in the solution set of the problem

$$
\min _{d_{1}+\cdots+d_{n}=d} \sum_{i=1}^{n}\left(X_{i}^{c}(\omega)-d_{i}\right)_{+},
$$

for all $\omega \in \Omega$, where $\mathbf{X}^{c}=\left(F_{X_{1}}^{-1}(U), \ldots, F_{X_{n}}^{-1}(U)\right)$ and $U$ is a uniform $(0,1)$ random variable.

This observation leads to the following generalization of Problem (7) in terms of a risk measure.

Proposition 3.3. Let $\rho$ be a risk measure which is (i) law invariant, (ii) increasing in the sense that $Z_{1} \leq$ st $Z_{2}$ (that is, $\mathbb{P}\left(Z_{1} \leq t\right) \geq \mathbb{P}\left(Z_{2} \leq t\right.$ ) for all $t$ ) implies that $\rho\left(Z_{1}\right) \leq \rho\left(Z_{2}\right)$, and (iii) comonotonic additive. For any $d \in \mathbb{R}$, the solution set of the problem

$$
\min _{d_{1}+\cdots+d_{n}=d} \sum_{i=1}^{n} \rho\left(\left(X_{i}-d_{i}\right)_{+}\right)
$$

contains $i\left(d, \mathbf{X}^{c}\right)$. 
Proof: By law-invariance and comonotonic additivity, the objection function in Problem (9) equals

$$
\rho\left(\sum_{i=1}^{n}\left(F_{X_{i}}^{-1}(U)-d_{i}\right)_{+}\right),
$$

where $U$ is a uniform $(0,1)$ random variable. Since $\sum_{i=1}^{n}\left(F_{X_{i}}^{-1}(U(\omega))-d_{i}\right)_{+}$is minimized under the constraint $\sum_{i} d_{i}=d$ on the set $i\left(d, \mathbf{X}^{c}\right)$ for all $\omega \in \Omega$, so in view of the monotonicity of $\rho$, we conclude that the solution set of Problem (9) contains $i\left(d, \mathbf{X}^{c}\right)$.

\section{Representing convex functions as stop-loss transforms}

In this section, we show that Problem (1) can be transformed into Problem (7) for some suitable random variables $X_{1}, \ldots, X_{n}$, and hence the solution set of Problem (1) equals $i\left(d, \mathbf{X}^{c}\right)$ by Theorem 3.1.

Recall that each $f_{i}$ in Problem (1) is assumed to be convex and continuous on $\left[l_{i}, u_{i}\right]$, with finite right-hand derivative at $l_{i}$ and finite left-hand derivative at $u_{i}$.

Proposition 4.1. Let $g:[l, u] \rightarrow \mathbb{R}$ be a continuous and convex function with

$$
-1 \leq g_{+}^{\prime}(l) \leq g_{-}^{\prime}(u) \leq 1 .
$$

Then there exists a random variable $X$ with $\mathbb{P}(l \leq X \leq u)=1$ such that

$$
g(x)=g(l)+x+l+2 \mathbb{E}\left[(X-x)_{+}\right]-2 \mathbb{E}(X) \text { for any } x \in[l, u] .
$$

Moreover, the distribution function of $X$ is given by

$$
F_{X}(x)= \begin{cases}0 & \text { if } \quad x<l, \\ \left(1+g_{+}^{\prime}(x)\right) / 2 & \text { if } \quad l \leq x<u, \\ 1 & \text { if } \quad x \geq u .\end{cases}
$$

Proof: By convexity, $g_{+}^{\prime}(x)$ is right-continuous and increasing on $[l, u)$. Condition (10) ensures that the expression in (11) is a genuine distribution function. We denote by $X$ an arbitrary random variable with such a distribution function. It is clear that $\mathbb{P}(l \leq X \leq u)=1$, and there is a possible jump at $l$ and at $u$. For any $x \in[l, u]$, we have

$$
\begin{aligned}
x & +2 \mathbb{E}\left[(X-x)_{+}\right]-2 \mathbb{E}(X) \\
& =\mathbb{E}|X-x|-\mathbb{E}(X) \\
& =\int_{0}^{x-l} F_{X-l}(t) d t+\int_{x-l}^{u-l}\left(1-F_{X-l}(t)\right) d t-\int_{0}^{u-l}\left(1-F_{X-l}(t)\right) d t-l \\
& =g(x)-g(l)-l .
\end{aligned}
$$

Rearranging this equation yields the desired result.

By the same argument, we have the following variant of Proposition 4.1, in which the upper end point $u$ of the domain of $g$ is infinity: 
Proposition 4.2. Let $g:[l, \infty) \rightarrow \mathbb{R}$ be a decreasing convex function with

$$
-1 \leq g_{+}^{\prime}(l) \leq \lim _{x \rightarrow \infty} g_{+}^{\prime}(x)=0 .
$$

Let $L:=\lim _{x \rightarrow \infty} g(x) \in \mathbb{R}$. Then there exists a random variable with $\mathbb{P}(X \geq l)=1$ such that

$$
g(x)=L+\mathbb{E}\left[(X-x)_{+}\right] \text {for any } x \geq l .
$$

Moreover, the distribution function of $X$ is given by

$$
F_{X}(x)=\left\{\begin{array}{lll}
0 & \text { if } & x<l \\
1+g_{+}^{\prime}(x) & \text { if } & x \geq l .
\end{array}\right.
$$

A common requirement in these two propositions is that the convex function concerned has bounded derivative on the relevant domain. Such requirement enables us to rescale linearly the right-hand derivative into a distribution function of a random variable. Our methodology remains valid even if the derivative is not bounded. In that case, instead of rescaling the right-hand derivative into a distribution function of a random variable, we can directly treat the right-hand derivative as the distribution function of a Radon measure on $\mathbb{R}$, see page 16 of Föllmer and Schied (2004) and page 545 of Revuz and Yor (1999). Comonotonicity of real-valued measurable maps on a general measurable space can be defined in exactly the same way as it is defined for random variables on a probability space, Property (4) on the additivity of the inverse distribution functions of comonotonic sums is also valid. We choose not to pursue such generality in order to put the focus on the ideas and techniques rather than on technicalities. Interested readers can easily work out the details for the general case.

\section{Solution set of minimization Problem (1)}

Now we return to our optimal capital allocation Problem (1):

$$
\min _{\mathbf{d} \in \mathcal{A}(d)} \sum_{i=1}^{n} f_{i}\left(d_{i}\right)
$$

where the functions $f_{i}$ are convex and continuous on $\left[l_{i}, u_{i}\right]$ with $-\infty<\left(f_{i}\right)_{+}^{\prime}\left(l_{i}\right) \leq\left(f_{i}\right)_{-}^{\prime}\left(u_{i}\right)<$ $\infty$, and

$$
\mathcal{A}(d)=\left\{\left(d_{1}, \ldots, d_{n}\right) \in \mathbb{R}^{n} \mid d_{1}+\cdots+d_{n}=d, d_{i} \in\left[l_{i}, u_{i}\right], i=1, \ldots, n\right\}
$$

is the set of admissible allocations with $l_{1}+\cdots+l_{n}<d<u_{1}+\cdots+u_{n}$. Since condition (10) of Proposition 4.1 may not be satisfied by $f_{i}$, a simple rescaling is needed. To this end, take $\nu$ to be any number that is strictly larger than $\nu^{*}$, which is defined by

$$
\nu^{*}:=\max _{1 \leq i \leq n}\left(\left|\left(f_{i}\right)_{+}^{\prime}\left(l_{i}\right)\right| \vee\left|\left(f_{i}\right)_{-}^{\prime}\left(u_{i}\right)\right|\right) \in \mathbb{R},
$$

and define the functions

$$
\tilde{f}_{i}(x):=f_{i}(x) / \nu \quad \text { for } x \in\left[l_{i}, u_{i}\right] \text { and } i=1, \ldots, n .
$$


The functions $\tilde{f}_{i}$ satisfy all conditions of Proposition 4.1, and hence there exist random variables $X_{1}, \ldots, X_{n}$ such that for $i=1, \ldots, n$,

$$
\tilde{f}_{i}(x)=\tilde{f}_{i}\left(l_{i}\right)+x+l_{i}+2 \mathbb{E}\left[\left(X_{i}-x\right)_{+}\right]-2 \mathbb{E}\left(X_{i}\right), \quad x \in\left[l_{i}, u_{i}\right]
$$

where the distribution function of $X_{i}$ is given by

$$
F_{X_{i}}(x)=\left\{\begin{array}{lll}
0 & \text { if } \quad x<l_{i}, \\
\left(1+\left(\tilde{f}_{i}\right)_{+}^{\prime}(x) / \nu\right) / 2 & \text { if } \quad l_{i} \leq x<u_{i}, \\
1 & \text { if } \quad x \geq u_{i} .
\end{array}\right.
$$

Moreover, as $\nu$ is chosen to be larger than $\nu^{*}$, each $X_{i}$ has a point mass at both of its essential infimum $F_{X_{i}}^{-1+}(0)=l_{i}$ and essential supremum $F_{X_{i}}^{-1}(1)=u_{i}$. With the above transformation, we find

$$
\sum_{i=1}^{n} f_{i}\left(d_{i}\right)=C+2 \nu \sum_{i=1}^{n} \mathbb{E}\left[\left(X_{i}-d_{i}\right)_{+}\right]
$$

for any $\left(d_{1}, \ldots, d_{n}\right) \in \mathcal{A}(d)$, where $C$ is some constant which is independent of $\left(d_{1}, \ldots, d_{n}\right)$. Therefore, Problem (1) is equivalent to the following problem:

$$
\min _{\mathbf{d} \in \mathcal{A}(d)} \sum_{i=1}^{n} \mathbb{E}\left[\left(X_{i}-d_{i}\right)_{+}\right]
$$

in the sense that the two problems have the same solution sets.

One immediately notices the similarity between Problem (7) and Problem (15). The only difference between them is that Problem (15) requires that $d_{i} \in\left[l_{i}, u_{i}\right]$ for all $i$ while Problem (7) does not. However, from Corollary 3.2 and (14), we know that any solution $\left(d_{1}^{*}, \ldots, d_{n}^{*}\right)$ of Problem (7) satisfies $d_{i}^{*} \in\left[F_{X_{i}}^{-1+}(0), F_{X_{i}}^{-1}(1)\right]=\left[l_{i}, u_{i}\right]$, so the box constraints on the individual allocations is automatically fulfilled. Combining this observation with Theorem 3.1 leads to following result:

Theorem 5.1. The solution set of Problem (1) equals $i\left(d, \mathbf{X}^{c}\right)$, where the marginal distribution of $X_{i}$ is given by (14). Moreover, if $d \in s\left(\mathbf{X}^{c}\right)$, the Problem (1) admits a unique solution.

In the remainder of this section, $S^{c}$ denotes the comonotonic sum $X_{1}^{c}+\cdots+X_{n}^{c}$, where $\left(X_{1}^{c}, \ldots, X_{n}^{c}\right)$ is a comonotonic modification of $\left(X_{1}, \ldots, X_{n}\right)$ with marginal distributions given by $(14)$.

Corollary 5.2. The solution set to Problem (1) is given by

$$
\left\{\left(d_{1}, \ldots, d_{n}\right) \mid d_{1}+\cdots+d_{n}=d, F_{X_{i}}^{-1}\left(F_{S^{c}}(d)\right) \leq d_{i} \leq F_{X_{i}}^{-1+}\left(F_{S^{c}}(d)\right) \text { for all } i\right\} .
$$

Moreover, $0<F_{S^{c}}(d)<1$.

Proof: $\quad$ Since $\nu$ is chosen to be strictly larger $\nu^{*}$ defined in (13), $F_{X_{i}}^{-1+}(0)=l_{i}$ and $F_{X_{i}}^{-1}(1)=$ $u_{i}$ for each $i$ and so $F_{S^{c}}^{-1+}(0)=\sum_{i} l_{i}<d<\sum_{i} u_{i}=F_{S^{c}}^{-1}(1)=n d$. This implies that $0<F_{S^{c}}(d)<1$. Now the result follows from Theorem 5.1 and Proposition 2.1. 
Corollary 5.3. Problem (1) has a unique solution given by $\left(F_{X_{1}}^{-1}\left(F_{S^{c}}(d)\right), \ldots, F_{X_{n}}^{-1}\left(F_{S^{c}}(d)\right)\right)$ if each $f_{i}$ is strictly convex.

Although this corollary is a standard result in the theory of convex minimization, here we will give a new and simple proof by using the theory of comonotonicity.

Proof: When each $f_{i}$ is strictly convex, each $F_{X_{i}}$ defined in (14) is strictly increasing on $\left[l_{i}, u_{i}\right]$, and hence $F_{X_{i}}^{-1}$ does not contain any discontinuity. In particular, this implies that $F_{X_{i}}^{-1}\left(F_{S^{c}}(d)\right)=F_{X_{i}}^{-1+}\left(F_{S^{c}}(d)\right)$ and so by Corollary 5.2, Problem (1) has a unique solution given by $\left(F_{X_{1}}^{-1}\left(F_{S}(d)\right), \ldots, F_{X_{n}}^{-1}\left(F_{S}(d)\right)\right)$.

The next result can be found in Bitran and Hax (1981). Instead of proving it using KuhnTucker theory, we demonstrate that it is a direct consequence of Corollary 5.2.

Corollary 5.4. Suppose that $f_{i}$ is strictly increasing on $\left[l_{i}, u_{i}\right]$ for $i \in J_{I} \subset\{1, \ldots, n\}$ and is strictly decreasing on $\left[l_{i}, u_{i}\right]$ for $i \in J_{D} \subset\{1, \ldots, n\}$. If $\left(d_{1}^{*}, \ldots, d_{n}^{*}\right)$ is a solution of Problem (1), then either $d_{i}^{*}=l_{i}$ for all $i \in J_{I}$, or $d_{i}^{*}=u_{i}$ for all $i \in J_{D}$, or both.

Proof: If $f_{i}$ is strictly increasing on $\left[l_{i}, u_{i}\right]$, the corresponding $F_{X_{i}}$ in (14) jumps at $l_{i}$ from 0 to $F_{X_{i}}\left(l_{i}\right)>1 / 2$, and hence $F_{X_{i}}^{-1}(p)=F_{X_{i}}^{-1+}(p)=l_{i}$ on an interval containing $K_{I} \supset(0,1 / 2]$. Similarly, if $f_{i}$ is strictly decreasing on $\left[l_{i}, u_{i}\right]$, the corresponding $F_{X_{i}}$ in (14) jumps at $u_{i}$ from $F_{X_{i}}\left(u_{i}-\right)<1 / 2$ to 1 , and hence $F_{X_{i}}^{-1}(p)=F_{X_{i}}^{-1+}(p)=u_{i}$ on an interval containing $K_{D} \supset[1 / 2,1)$. From Corollary 5.2, if $F_{S^{c}}(d) \in K_{I}$, then $d_{i}^{*}=l_{i}$ for all $i \in J_{\mathrm{I}}$; if $F_{S^{c}}(d) \in K_{D}$, then $d_{i}^{*}=u_{i}$ for all $i \in J_{\mathrm{D}}$. As $K_{I} \cup K_{D}=(0,1)$, the result follows.

\section{$6 \quad$ Examples}

In this section, we provide three examples to illustrate how the theory developed above can be used to solve practically relevant problems.

Example 1 Let $X_{i}, i=1, \ldots, n$ be positive and integrable risks. We consider the following optimal capital allocation problem, which is a special case of Problem (9) studied in Section 3:

$$
\min _{d_{1}+\cdots+d_{n}=d, d_{i} \geq 0} \sum_{i=1}^{n} \operatorname{TVaR}_{\alpha}\left(\left(X_{i}-d_{i}\right)_{+}\right), \quad d>0 .
$$

Here, $\operatorname{TVaR}_{\alpha}$ denotes the Tail Value-at-Risk at probability level $\alpha \in(0,1)$ which is defined by

$$
\operatorname{TVaR}_{\alpha}(X):=\frac{1}{1-\alpha} \int_{\alpha}^{1} F_{X}^{-1}(p) d p
$$

for any integrable random variable $X$. Since Tail Value-at-Risk is law-invariant, increasing, and comonotonic additive, it follows from Proposition 3.3 that the solution set of Problem (16) contains $i\left(d, \mathbf{X}^{c}\right)$. Our objective here is to derive this result by using the methodology developed in Sections 4 and 5.

To this end, we first define $f_{i}\left(d_{i}\right):=\operatorname{TVaR}_{\alpha}\left(\left(X_{i}-d_{i}\right)_{+}\right)$for $i=1, \ldots, n$. Each $f_{i}:[0, \infty) \rightarrow$ 
$\mathbb{R}$ is a decreasing and convex function, and it is straightforward to obtain

$$
f_{i}^{\prime}\left(d_{i}\right)=\left\{\begin{array}{lll}
-1 & \text { if } & d_{i}<F_{X_{i}}^{-1}(\alpha) \\
-\frac{1-F_{R_{i}}\left(d_{i}\right)}{1-\alpha} & \text { if } & d_{i} \geq F_{X_{i}}^{-1}(\alpha)
\end{array}\right.
$$

So it follows from Proposition 4.2 that we can express $f_{i}$ as

$$
f_{i}\left(d_{i}\right)=\mathbb{E}\left[\left(Y_{i}-d_{i}\right)_{+}\right], \quad d_{i} \geq 0,
$$

where the distribution function of $Y_{i}$ is given by

$$
F_{Y_{i}}(x)=\left\{\begin{array}{lll}
0 & \text { if } & x<F_{X_{i}}^{-1}(\alpha) \\
\frac{F_{X_{i}}(x)-\alpha}{1-\alpha} & \text { if } & x \geq F_{X_{i}}^{-1}(\alpha) .
\end{array}\right.
$$

Using this transformation, Problem (16) becomes

$$
\min _{d_{1}+\cdots+d_{n}=d, d_{i} \geq 0} \sum_{i=1}^{n} \mathbb{E}\left[\left(Y_{i}-d_{i}\right)_{+}\right]
$$

and hence Corollary 5.2 implies that the solution set of Problem (16) is given by $i\left(d, \mathbf{Y}^{c}\right)$ :

$$
\left\{\left(d_{1}, \ldots, d_{n}\right) \mid d_{1}+\cdots+d_{n}=d, F_{Y_{i}}^{-1}\left(F_{S_{Y}^{c}}(d)\right) \leq d_{i} \leq F_{Y_{i}}^{-1+}\left(F_{S_{Y}^{c}}(d)\right) \text { for all } i\right\},
$$

in which $S_{Y}^{c}:=Y_{1}^{c}+\cdots+Y_{n}^{c}$ and $\left(Y_{1}^{c}, \ldots, Y_{n}^{c}\right)$ is a comonotonic copy of $\left(Y_{1}, \ldots, Y_{n}\right)$. From relationship (17), which is piecewise linear, it is easy to express $F_{Y_{i}}^{-1}$ in terms of $F_{X_{i}}^{-1}$, and $F_{S_{Y}^{c}}$ in terms of $F_{S_{X}^{c}}$ where $S_{X}^{c}$ denotes the corresponding sum of a comonotonic copy of $\left(X_{1}, \ldots, X_{n}\right)$. We then can easily show that $i\left(d, \mathbf{Y}^{c}\right)$ is identical to $i\left(d, \mathbf{X}^{c}\right)$.

Example 2 Consider the following optimal capital allocation problem:

$$
\min _{d_{1}+\cdots+d_{n}=d, d_{i} \geq l_{i}} \sum_{i=1}^{n} s_{i} \exp \left(-m_{i} d_{i}\right)
$$

where $s_{i}, m_{i}$ are some strictly positive constants. Notice that $d_{i} \mapsto-s_{i} \exp \left(-m_{i} d_{i}\right)$ is a standard utility function with constant absolute risk aversion. It is assumed that

$$
d>L:=l_{1}+\cdots+l_{n}
$$

in order to avoid that the problem is trivial or ill-posed. To simplify the notation, we define

$$
\theta_{i}:=\frac{\ln m_{i} s_{i}}{m_{i}}, \quad \Theta_{i}:=\sum_{j=1}^{i} \theta_{j}, \quad A_{i}:=1-m_{i} s_{i} \exp \left(-m_{i} l_{i}\right), \quad M_{i}:=\sum_{j=1}^{i} \frac{1}{m_{j}}, \quad L_{i}:=\sum_{j=i+1}^{n} l_{j},
$$

for $i=1, \ldots, n$. Note that $L_{n}:=0$ by convention. Without loss of generality, we assume that $A_{1} \leq \cdots \leq A_{n}$.

Proposition 6.1. For any given $d>L$, define

$$
i^{*}:=\inf \left\{i \in\{1, \ldots, n-1\} \mid d \leq L+\sum_{j=1}^{i} M_{j}\left(\ln \left(1-A_{j}\right)-\ln \left(1-A_{j+1}\right)\right)\right\} \wedge n,
$$

with the convention that inf $\emptyset=\infty$. Then the solution to Problem (19) is given by

$$
d_{i}^{*}= \begin{cases}l_{i}, & i=i^{*}+1, \ldots, n, \\ \theta_{i}-\frac{\Theta_{i^{*}}+L_{i^{*}}-d}{m_{i} M_{i^{*}}}, & i=1, \ldots, i^{*}\end{cases}
$$


Proof: We first notice that every $f_{i}\left(d_{i}\right):=s_{i} \exp \left(-m_{i} d_{i}\right)$ is strictly decreasing and strictly convex. Without loss of generality, we may assume that $s_{1}, \ldots, s_{n}$ have been rescaled properly such that $-1 \leq\left(f_{i}\right)_{+}^{\prime}\left(l_{i}\right)$ for all $i$. Since $\lim _{x \rightarrow \infty} f_{i}(x)$ exists in $\mathbb{R}$ and $\lim _{x \rightarrow \infty}\left(f_{i}\right)_{+}^{\prime}(x)=0$ for all $i$, it follows from Proposition 4.2 and Corollary 5.3 that Problem (19) has a unique solution $\left(d_{1}^{*}, \ldots, d_{n}^{*}\right)$ given by $d_{i}^{*}=F_{X_{i}}^{-1}\left(F_{S^{c}}(d)\right)$ where $S^{c}:=F_{X_{1}}^{-1}(U)+\cdots+F_{X_{n}}^{-1}(U)$ for any uniform $(0,1)$ random variable $U$ and

$$
F_{X_{i}}(x)= \begin{cases}0, & x<l_{i} \\ 1-m_{i} s_{i} \exp \left(-m_{i} x\right), & l_{i} \leq x\end{cases}
$$

It remains to compute $F_{S^{c}}(d)$ and $F_{X_{i}}^{-1}$.

The inverse of this distribution function is given by

$$
F_{X_{i}}^{-1}(p)=\left\{\begin{array}{ll}
l_{i}, & 0<p \leq A_{i} \\
\frac{1}{m_{i}} \ln \frac{m_{i} s_{i}}{1-p}, & A_{i} \leq p<1,
\end{array}=\left(\frac{1}{m_{i}} \ln \frac{m_{i} s_{i}}{1-p}\right) \vee l_{i} .\right.
$$

Also, for any $p \in(0,1)$, we find that

$$
\begin{aligned}
F_{S^{c}}^{-1}(p)= & F_{X_{1}}^{-1}(p)+\cdots+F_{X_{n}}^{-1}(p) \\
& \begin{cases}l_{1}+\cdots+l_{n}, & 0<p \leq A_{1}, \\
\frac{1}{m_{1}} \ln \frac{m_{1} s_{1}}{1-p}+l_{2}+\cdots+l_{n}, & A_{1} \leq p \leq A_{2}, \\
\frac{1}{m_{1}} \ln \frac{m_{1} s_{1}}{1-p}+\frac{1}{m_{2}} \ln \frac{m_{2} s_{2}}{1-p}+l_{3}+\cdots+l_{n}, & A_{2} \leq p \leq A_{3}, \\
\vdots & \\
\frac{1}{m_{1}} \ln \frac{m_{1} s_{1}}{1-p}+\cdots+\frac{1}{m_{n}} \ln \frac{m_{n} s_{n}}{1-p}, & A_{n} \leq p<A_{n+1}:=1 .\end{cases}
\end{aligned}
$$

Notice that $F_{S^{c}}^{-1}$ is continuous and is piecewise linear on $\left(0, A_{1}\right],\left[A_{1}, A_{2}\right], \ldots,\left[A_{n}, A_{n+1}\right)$. Simple algebraic manipulation shows that

$$
F_{S^{c}}^{-1}\left(A_{i+1}\right)=L+\sum_{j=1}^{i} M_{j}\left(\ln \left(1-A_{j}\right)-\ln \left(1-A_{j+1}\right)\right), \quad i=1, \ldots, n-1,
$$

which is the expression in (21). The definition of $i^{*}$ in (21) enables us to locate the exact "layer" that $F_{S^{c}}(d)$ belongs to, so that $A_{i^{*}}<F_{S^{c}}(d) \leq A_{i^{*}+1}$. By solving the equation $F_{S^{c}}^{-1}\left(F_{S^{c}}(d)\right)=d$ for $F_{S^{c}}(d)$ using this particular layer in $(22)$, we obtain

$$
F_{S^{c}}(d)=1-\exp \left(\frac{\Theta_{i^{*}}+L_{i^{*}}-d}{M_{i^{*}}}\right), \quad d>L .
$$

Therefore, the solution to Problem (19) is given by

$$
d_{i}^{*}=F_{X_{i}}^{-1}\left(F_{S^{c}}(d)\right)=\left(\frac{1}{m_{i}} \ln \frac{m_{i} s_{i}}{1-F_{S^{c}}(d)}\right) \vee l_{i}, \quad i=1, \ldots, n .
$$

Simplifying this expression yields the desired result.

For instance, if

$$
L<d \leq \frac{1}{m_{1}} \ln \frac{m_{1} s_{1}}{1-A_{2}}+l_{2}+\cdots+l_{n}
$$


then $i^{*}=1$ and $A_{1}<F_{S^{c}}(d) \leq A_{2}$. Therefore, the solution is given by

$$
d_{i}^{*}= \begin{cases}\theta_{1}-\frac{\Theta_{1}+L_{1}-d}{m_{1} M_{1}}=d-\left(l_{2}+\cdots+l_{n}\right), & i=1, \\ l_{i}, & i=2, \ldots, n .\end{cases}
$$

As another illustration, suppose that $d$ is sufficiently large such that

$$
d>\frac{1}{m_{1}} \ln \frac{m_{1} s_{1}}{1-A_{n}}+\cdots+\frac{1}{m_{n}} \ln \frac{m_{n} s_{n}}{1-A_{n}}=L+\sum_{j=1}^{n-1} M_{j}\left(\ln \left(1-A_{j}\right)-\ln \left(1-A_{j+1}\right)\right),
$$

then $i^{*}=n$ and $A_{n}<F_{S^{c}}(d)<1$. Applying Proposition 6.1 yields that

$$
d_{i}^{*}=\theta_{i}-\frac{\Theta_{n}-d}{m_{i} M_{n}}>l_{i}, \quad i=1, \ldots, n .
$$

Example 3 In Dhaene et al. (2012), the following optimal capital allocation was considered:

$$
\min _{d_{1}+\cdots+d_{n}=d} \sum_{i=1}^{n} \mathbb{E}\left[\frac{\zeta_{i}\left(Y_{i}-d_{i}\right)^{2}}{\nu_{i}}\right]
$$

where $\zeta_{1}, \ldots, \zeta_{n}$ are positive random variables with mean $1, \nu_{1}, \ldots, \nu_{n}$ are given strictly positive numbers summing to 1 , and $Y_{1}, \ldots, Y_{n}$ are some square integrable random variables. We refer to Dhaene et al. (2012) for a detailed interpretation of this model. In that paper, it is shown that the optimal allocations are given by

$$
d_{i}^{*}=\nu_{i}\left(d-\sum_{j=1}^{n} \mathbb{E}\left[\zeta_{j} Y_{j}\right]\right)+\mathbb{E}\left[\zeta_{i} Y_{i}\right], \quad i=1, \ldots, n .
$$

Here, we want to add the box constraints $d_{i} \in[0, d]$ for all $i$ to the minimization problem above. More precisely, we would like to apply the theory developed in the previous sections to solve the following problem:

$$
\min _{d_{1}+\cdots+d_{n}=d, 0 \leq d_{i} \leq d} \sum_{i=1}^{n} \mathbb{E}\left[\frac{\zeta_{i}\left(Y_{i}-d_{i}\right)^{2}}{\nu_{i}}\right] .
$$

To simplify our notation, we define $c_{i}:=\mathbb{E}\left[\zeta_{i} Y_{i}\right]$ for $i=1, \ldots, n$ and assume without loss of generality that

$$
\frac{c_{1}}{\nu_{1}} \geq \cdots \geq \frac{c_{n}}{\nu_{n}}
$$

Proposition 6.2. For any given $d>0$, define

$$
i^{*}:=\inf \left\{i \in\{1, \ldots, n-1\} \mid d \leq \sum_{j=1}^{i} \nu_{j}\left(\frac{c_{j}}{\nu_{j}}-\frac{c_{i+1}}{\nu_{i+1}}\right)\right\} \wedge n,
$$

with the convention that inf $\emptyset=\infty$. Then the solution to Problem (24) is given by

$$
d_{i}^{*}= \begin{cases}\frac{\nu_{i}}{\sum_{i=1}^{i^{*} \nu_{j}}}\left(d-\sum_{j=1}^{i^{*}} c_{j}\right)+c_{i}, & i=1, \ldots, i^{*} \\ 0, & i=i^{*}+1, \ldots, n .\end{cases}
$$


Proof: We first let

$$
f_{i}(x):=\mathbb{E}\left[\frac{\zeta_{i}\left(Y_{i}-x\right)^{2}}{\nu_{i}}\right], \quad x \in \mathbb{R}, i=1, \ldots, n .
$$

Notice that the box constraints $d_{i} \in[0, d]$ for all $i$ can be replaced by $d_{i} \in\left[0, u_{i}\right]$ for all $i$ as long as each $u_{i}$ is larger than $d$. In particular, we choose $u_{1}, \ldots, u_{n}$ greater than $d$ such that

$$
\left(f_{1}\right)_{+}^{\prime}\left(u_{1}\right)=\cdots=\left(f_{n}\right)_{+}^{\prime}\left(u_{n}\right) \geq \max _{1 \leq i \leq n}\left(f_{i}\right)_{+}^{\prime}(0),
$$

which is equivalent to

$$
\frac{2\left(u_{1}-\mathbb{E}\left[\zeta_{1} Y_{1}\right]\right)}{\nu_{1}}=\cdots=\frac{2\left(u_{n}-\mathbb{E}\left[\zeta_{n} Y_{n}\right]\right)}{\nu_{n}} \geq \max _{1 \leq i \leq n}\left|\frac{-2 \mathbb{E}\left[\zeta_{i} Y_{i}\right]}{\nu_{i}}\right|
$$

Moreover, we take $\nu$ to be the common value on the left-hand side of the inequality above. By Proposition 4.1, Problem (24) is equivalent to

$$
\min _{d_{1}+\cdots+d_{n}=d, 0 \leq d_{i} \leq u_{i}} \sum_{i=1}^{n} \mathbb{E}\left[\left(X_{i}-d_{i}\right)_{+}\right],
$$

where the distribution function of $X_{i}$ is given by

$$
F_{X_{i}}(x)= \begin{cases}0 & \text { if } \quad x<0 \\ \left(1+f_{+}^{\prime}(x) / \nu\right) / 2 & \text { if } \quad 0 \leq x<u_{i} \\ 1 & \text { if } \quad x \geq u_{i}\end{cases}
$$

Our choice of $u_{1}, \ldots, u_{n}$ guarantees that none of the distribution function $F_{X_{i}}$ has a point mass at $u_{i}$. Inverting the distribution function above yields that

$$
F_{X_{i}}^{-1}(p)=\left\{\begin{array}{ll}
0, & 0<p \leq A_{i} \\
\nu \nu_{i}(p-1 / 2)+c_{i}, & A_{i} \leq p<1,
\end{array}=\left(\nu \nu_{i}(p-1 / 2)+c_{i}\right)_{+}\right.
$$

where $A_{i}:=1 / 2-\frac{c_{i}}{\nu \nu_{i}}$ for $i=1, \ldots, n$. From assumption (25), $A_{1} \leq \cdots \leq A_{n}$. Let $S^{c}$ be the comonotonic sum $F_{X_{1}}^{-1}(U)+\cdots+F_{X_{n}}^{-1}(U)$, where $U$ is any uniform $(0,1)$ random variable. Then for $p \in(0,1)$,

$$
\begin{aligned}
F_{S^{c}}^{-1}(p) & =F_{X_{1}}^{-1}(p)+\cdots+F_{X_{n}}^{-1}(p) \\
& = \begin{cases}0, & 0<p \leq A_{1}, \\
\nu \nu_{1}(p-1 / 2)+c_{1}, & A_{1} \leq p \leq A_{2}, \\
\nu \nu_{1}(p-1 / 2)+c_{1}+\nu \nu_{2}(p-1 / 2)+c_{2}, & A_{2} \leq p \leq A_{3}, \\
\vdots & \\
\nu \nu_{1}(p-1 / 2)+c_{1}+\cdots+\nu \nu_{n}(p-1 / 2)+c_{n}, & A_{n} \leq p<1 .\end{cases}
\end{aligned}
$$

By a similar argument as in the proof of Proposition 6.1, we obtain

$$
F_{S^{c}}(d)=\frac{1}{2}+\frac{d-\sum_{j=1}^{i^{*}} c_{j}}{\nu \sum_{i=1}^{i^{*}} \nu_{j}}
$$


From Corollary 5.3, the solution to Problem (24) is given by

$$
d_{i}^{*}=F_{X_{i}}^{-1}\left(F_{S^{c}}(d)\right)=\left(\nu \nu_{i}\left(F_{S^{c}}(d)-1 / 2\right)+c_{i}\right)_{+}, \quad i=1, \ldots, n .
$$

Putting the expression of $F_{S^{c}}(d)$ in (27) in this formula yields the desired result.

As an illustration, consider the case where

$$
0<d \leq \nu_{1}\left(\frac{c_{1}}{\nu_{1}}-\frac{c_{2}}{\nu_{2}}\right)
$$

Then $i^{*}=1$ and $d_{1}^{*}=d$ and $d_{2}^{*}=\cdots=d_{n}^{*}=0$. As another illustration, if

$$
\sum_{j=1}^{n-1} \nu_{j}\left(\frac{c_{j}}{\nu_{j}}-\frac{c_{n}}{\nu_{n}}\right)<d
$$

then $i^{*}=n$ and

$$
d_{i}^{*}=\nu_{i}\left(d-\sum_{j=1}^{n} c_{j}\right)+c_{i}, \quad i=1, \ldots, n,
$$

which is the solution obtained in Dhaene et al. (2012) without any box constraints.

\section{Some variants}

\subsection{Minimization of a weighted sum of stop-loss premiums}

Consider the following variant of minimization Problem (7):

$$
\min _{d_{1}+\cdots+d_{n}=d, l_{i} \leq d_{i} \leq u_{i}} \sum_{i=1}^{n} \nu_{i} \mathbb{E}\left[\left(Y_{i}-d_{i}\right)_{+}\right],
$$

where $\nu_{1}, \ldots, \nu_{n}$ are some strictly positive constants that are not all equal, and $Y_{1}, \ldots, Y_{n}$ are some integrable random variables with possibly unbounded support.

We first remark that the support of each $Y_{i}$ in Problem (28) can be assumed to be contained in $\left[l_{i}, u_{i}\right]$ without loss of generality. For if $d_{i} \in\left[l_{i}, u_{i}\right]$,

$$
\mathbb{E}\left[\left(Y_{i}-d_{i}\right)_{+}\right]=\mathbb{E}\left[\left(Y_{i} \vee l_{i}-d_{i}\right)_{+}\right]=\mathbb{E}\left[\left(\left(Y_{i} \vee l_{i}\right) \wedge u_{i}-d_{i}\right)_{+}\right]+\mathbb{E}\left[\left(Y_{i}-u_{i}\right)_{+}\right],
$$

and thus $Y_{i}$ can be replaced by $\left(Y_{i} \vee l_{i}\right) \wedge u_{i}$ in Problem (28) without changing the solution set. For the remainder of this section, we assume that the support of each $Y_{i}$ is contained in $\left[l_{i}, u_{i}\right]$.

To solve Problem (28), one may simply treat it as a special case of Problem (1) by writing

$$
f_{i}\left(d_{i}\right):=\nu_{i} \mathbb{E}\left[\left(Y_{i}-d_{i}\right)_{+}\right], \quad d_{i} \in\left[l_{i}, u_{i}\right],
$$

and proceed as in Section 4 to express $f_{i}\left(d_{i}\right)$ as an affine function of $\mathbb{E}\left[\left(X_{i}-d_{i}\right)_{+}\right]$for some suitable $X_{i}$ so that the leading coefficients are equalized. 
In what follows, we present a simple trick to accomplish this transformation by using suitable Bernoulli variables to "absorb" the coefficients $\nu_{i}$. To explain this approach, we first assume, without loss of generality, that each $\nu_{i}$ is strictly less than 1 . If not, we may simply replace $\nu_{i}$ by $\nu_{i} / \sum \nu_{i}$. Let $Z_{1}, \ldots, Z_{n}$ be Bernoulli variables which are independent of $Y_{1}, \ldots, Y_{n}$ so that

$$
Z_{i}=\left\{\begin{array}{l}
1 \quad \text { with probability } \nu_{i}, \\
0 \quad \text { with probability } 1-\nu_{i},
\end{array}\right.
$$

and $X_{i}:=Z_{i}\left(Y_{i}-l_{i}\right)+l_{i}$. Then for any $l_{i} \leq d_{i} \leq u_{i}$,

$$
\begin{aligned}
\nu_{i} \mathbb{E}\left[\left(Y_{i}-d_{i}\right)_{+}\right] & =\mathbb{P}\left(Z_{i}=1\right) \mathbb{E}\left[\left(\left(Y_{i}-l_{i}\right)-\left(d_{i}-l_{i}\right)\right)_{+}\right] \\
& =\mathbb{E}\left[\left(Z_{i}\left(Y_{i}-l_{i}\right)-\left(d_{i}-l_{i}\right)\right)_{+}\right] \\
& =\mathbb{E}\left[\left(X_{i}-d_{i}\right)_{+}\right]
\end{aligned}
$$

by the assumed independence between $Z_{i}$ and $X_{i}$.

\subsection{Nonlinear constraints}

In Problem (1), the linear constraint $d_{1}+\cdots+d_{n}=d$ can be replaced by a non-linear constraint of the form

$$
h_{1}\left(d_{1}\right)+\cdots+h_{n}\left(d_{n}\right)=d,
$$

where each $h_{i}$ is a $1-1$ function so that the inverse $h_{i}^{-1}$ is well-defined (on a suitable domain). Let $\tilde{d}_{i}:=h_{i}\left(d_{i}\right)$ and $\tilde{f}_{i}(x)=f_{i}\left(h_{i}^{-1}(x)\right)$ for all $i$. If each $h_{i}^{-1}$ is convex and $f_{i}$ is increasing and convex, or $h_{i}^{-1}$ is concave and $f_{i}$ is decreasing and convex, then $\tilde{f}_{i}$ is convex as well, and hence the corresponding minimization problem is reduced to the form of Problem (1) again.

\section{Connection with infimum-convolution}

If the box constraints $d_{i} \in\left[l_{i}, u_{i}\right]$ for all $i$ in Problem (1) are removed (but $d_{1}+\cdots+d_{n}=d$ is kept), and the domain of each real-valued convex function $f_{i}$ is $\mathbb{R}$ instead of $\left[l_{i}, u_{i}\right]$, then

$$
\left(\wedge_{i=1}^{n} f_{i}\right)(d):=\min _{d_{1}+\cdots+d_{n}=d} \sum_{i=1}^{n} f_{i}\left(d_{i}\right)
$$

is called the infimum-convolution of $f_{1}, \ldots, f_{n}$ in convex analysis (cf. Rockafellar (1970)). Notice that the minimization in (29) may not have a solution in general.

Proposition 8.1. Consider some $\left(d_{1}^{*}, \ldots, d_{n}^{*}\right)$ with $d_{1}^{*}+\cdots+d_{n}^{*}=d$. Then

$$
\left(\wedge_{i=1}^{n} f_{i}\right)(d)=\sum_{i=1}^{n} f_{i}\left(d_{i}^{*}\right)
$$

if and only if

$$
\partial f_{1}\left(d_{1}^{*}\right) \cap \cdots \cap \partial f_{n}\left(d_{n}^{*}\right) \neq \emptyset
$$


Here, $\partial f_{i}\left(d_{i}\right):=\left[\left(f_{i}\right)_{-}^{\prime}\left(d_{i}\right),\left(f_{i}\right)_{+}^{\prime}\left(d_{i}\right)\right]$ is the subdifferential of $f_{i}$ at $d_{i}$. The objective here is to prove this result using the perspective of comonotonicity and the theory we developed in previous sections.

Proof: We first prove the "if" part. Fix some $\left(d_{1}^{*}, \ldots, d_{n}^{*}\right)$ with $d_{1}^{*}+\cdots+d_{n}^{*}=d$ and some $u^{*}$ such that

$$
u^{*} \in \partial f_{1}\left(d_{1}^{*}\right) \cap \cdots \cap \partial f_{n}\left(d_{n}^{*}\right) .
$$

Choose any $u_{1}, \ldots, u_{n}, l_{1}, \ldots, l_{n}$ such that $l_{i}<d_{i}^{*}<u_{i}$ for all $i$, and let $\mathbf{X}^{c}=\left(X_{1}^{c}, \ldots, X_{n}^{c}\right)$ be a comonotonic random vector with marginal distributions given by (14) for some large enough $\nu$. By $(14),\left(f_{i}\right)_{-}^{\prime}\left(d_{i}^{*}\right)=\nu\left(2 F_{X_{i}}\left(d_{i}^{*}-\right)-1\right)$ and $\left(f_{i}\right)_{+}^{\prime}\left(d_{i}^{*}\right)=\nu\left(2 F_{X_{i}}\left(d_{i}^{*}\right)-1\right)$, and so

$$
F_{X_{i}}\left(d_{i}^{*}-\right) \leq u^{* *} \leq F_{X_{i}}\left(d_{i}^{*}\right) \text { for all } i,
$$

where $u^{* *}:=\left(u^{*} / \nu+1\right) / 2$. This can be rewritten as

$$
F_{X_{i}}^{-1}\left(u^{* *}\right) \leq d_{i} \leq F_{X_{i}}^{-1+}\left(u^{* *}\right) \text { for all } i .
$$

In particular, this implies that $\left(d_{1}^{*}, \ldots, d_{n}^{*}\right) \in i\left(d, \mathbf{X}^{c}\right)$. By Theorem $5.1,\left(d_{1}^{*}, \ldots, d_{n}^{*}\right)$ solves the problem

$$
\min _{d_{1}+\cdots+d_{n}=d, l_{i} \leq d_{i} \leq u_{i}} \sum_{i=1}^{n} f_{i}\left(d_{i}\right) .
$$

However, as the $l_{i}$ 's and the $u_{i}$ 's can be chosen arbitrarily small and large respectively, we conclude that

$$
\min _{d_{1}+\cdots+d_{n}=d} \sum_{i=1}^{n} f_{i}\left(d_{i}\right)=\sum_{i=1}^{n} f_{i}\left(d_{i}^{*}\right) .
$$

This proves the "if" part.

For the "only if" part, suppose that $d_{1}^{*}+\cdots+d_{n}^{*}=d$ and $\left(\wedge_{i=1}^{n} f_{i}\right)(d)=\sum_{i=1}^{n} f_{i}\left(d_{i}^{*}\right)$. Choose any $u_{1}, \ldots, u_{n}, l_{1}, \ldots, l_{n}$ such that $l_{i}<d_{i}^{*}<u_{i}$ for all $i$. Then $\left(d_{1}^{*}, \ldots, d_{n}^{*}\right)$ solves the problem

$$
\min _{d_{1}+\cdots+d_{n}=d, l_{i} \leq d_{i} \leq u_{i}} \sum_{i=1}^{n} f_{i}\left(d_{i}\right) .
$$

It then follows from Theorem 5.1 and Proposition 2.1 that $F_{X_{i}}^{-1}\left(F_{S^{c}}(d)\right) \leq d_{i}^{*} \leq F_{X_{i}}^{-1+}\left(F_{S^{c}}(d)\right)$ for all $i$, where $\left(X_{1}^{c}, \ldots, X_{n}^{c}\right)$ is a comonotonic random vector with marginal distributions given by (14) for some large enough $\nu$, and $S^{c}:=X_{1}^{c}+\cdots+X_{n}^{c}$. From the proof of the "if" part, we have

$$
\nu\left(2 F_{S^{c}}(d)-1\right) \in \partial f_{1}\left(d_{1}^{*}\right) \cap \cdots \cap \partial f_{n}\left(d_{n}^{*}\right) .
$$

This shows that the right hand intersection is non-empty.

Proposition 8.1 is well-known. It gives necessary and sufficient conditions for $\left(d_{1}^{*}, \ldots, d_{n}^{*}\right)$ to be the solution of minimization problem (29).

\section{Acknowledgments}

Ka Chun Cheung acknowledges the financial support of the Research Grants Council of HKSAR (GRF Project 17324516). Jan Dhaene acknowledges the financial support of Onderzoeksfonds KU Leuven (GOA/13/002). Phillip Yam acknowledges the financial support from 
The Hong Kong RGC GRF 14301015 with the project title: Advance in Mean Field Theory and The Hong Kong RGC GRF 14300717 with the project title: New kinds of Forwardbackward Stochastic Systems with Applications. Phillip Yam also acknowledges the financial support from Department of Statistics of Columbia University in the City of New York during the period he was a visiting faculty member.

\section{References}

[1] Bitran, G.R., Hax, A.C., (1981). Disaggregation and resource allocation using convex knapsack problems with bounded variables. Management Science, 27, 431-441.

[2] Chen, X., Deelstra, G., Dhaene, J., Linders, D., Vanmaele, M., (2015). On an optimization problem related to static super-replicating strategies. Journal of Computational and Applied Mathematics, 278, 213-230

[3] Cheung, K.C., Rong, Y., Yam, S.C., (2014). Borch's Theorem from the perspective of comonotonicity. Insurance: Mathematics and Economics, 54, 144-151.

[4] Denneberg, D., (1994). Non-additive Measure and Integral. Kluwer Academic Publishers, Boston.

[5] Dhaene, J., Denuit, M., Goovaerts, M.J., Kaas, R., Vyncke, D., (2002). The concept of comonotonicity in actuarial science and finance: theory. Insurance: Mathematics and Economics, 31, 3-33.

[6] Dhaene, J., Tsanakas, A., Valdez, E., Vanduffel, S., (2012). Optimal capital allocation principles. Journal of Risk and Insurance, 79, 1-28.

[7] Föllmer, H, Schied, A., (2004). Stochastic Finance: An Introduction in Discrete Time, 2nd Edition. de Gruyter, Berlin.

[8] Frostig, E., Zaks, Y, Levikson, B., (2007). Optimal pricing for a heterogeneous portfolio for a given risk factor and convex distance measure. Insurance: Mathematics and Insurance, 40, 459-467.

[9] Kaas, R., Dhaene, J., Goovaerts, M., (2000). Upper and lower bounds for sums of random variables. Insurance: Mathematics and Economics, 27, 151-168.

[10] Kaas, R., Dhaene, J., Vyncke, D., Goovaerts, M., Denuit, M., (2002). A simple geometric proof that comonotonic risks have the convex-largest sum. ASTIN Bulletin, 32, 71-80.

[11] Luss, H., Gupta, S.K., (1975). Allocation of Effort Resources Among Competing Activities. Operations Research, 23, 360-366.

to the length of critical paths. Journal of Applied Probability, 16, 671-677.

[12] Revuz, D., Yor, D., (1999). Continuous Martingales and Brownian Motion, 3rd Edition. Springer, Berlin.

[13] Rockafellar, R., (1970). Convex Analysis. Princeton University Press, Princeton, N.J. 
[14] Rüschendorf, L., (2013). Mathematical Risk Analysis: Dependence, Risk Bounds, Optimal Allocations and Portfolios. Springer, Heidelberg.

[15] Stefanov, S., (2005). Convex separable minimization problems with a linear constraint and bounded variables. International Journal of Mathematics and Mathematical Sciences, 9, 1339-1363.

[16] Wilkinson, C., Gupta, S.K., (1969). Allocating Promotional Effort to Competing Activities: A Dynamic Programming Approach. IFORS Conference, Venice, 419-432.

[17] Zaks, Y, (2013). Optimal capital allocation - a generalization of the optimization problem. Insurance Markets and Companies: Analyses and Actuarial Computations International Research Journal, 4, 29-34.

[18] Zaks, Y, Frostig, E., Levikson, B., (2006). Optimal pricing of a heterogeneous portfolio for a given risk level. ASTIN Bulletin, 36, 161-185. 